\title{
PERSPECTIVAS DE INSERÇÃO DA ENERGIA SOLAR FOTOVOLTAICA NA GERAÇÃO DE ENERGIA ELÉTRICA NO RIO GRANDE DO NORTE
}

\author{
A. C. F. WANDERLEY ${ }^{1,2}$ e A. L. P. S. CAMPOS $^{2}$ \\ ${ }^{1}$ Diretoria Acadêmica de Indústria, Instituto Federal do Rio Grande do Norte (DIACIN/IFRN) \\ ${ }^{2}$ Programa de Pós-Graduação em Engenharia Elétrica e de Computação, Universidade Federal do Rio Grande do \\ Norte (PPGEEC/UFRN) \\ 1augusto.fialho@ifrn.edu.br - ²alpscampos@gmail.com
}

Artigo submetido em junho/2013 e aceito em julho/2013

\section{RESUMO}

A geração de energia elétrica no Brasil é predominantemente renovável, com a geração interna hidráulica sendo superior a $74 \%$ do total de sua matriz energética. $O$ racionamento de energia elétrica ocorrido em 2001, devido à falta de chuvas, levou o país a incrementar a participação de fontes alternativas de energia. Esta necessidade por novas fontes de energia faz com que as potencialidades regionais sejam aproveitadas, o que configura a mudança do modelo de geração, passando de geração centralizada para geração distribuída. Dentre as fontes alternativas de energia, a solar apresenta-se como uma fonte bastante promissora para o Brasil, tendo em vista que a maior parte do seu território está localizada próximo à linha do equador, o que acarreta em um dia de maior duração solar. O Rio Grande do Norte (RN) tem um dos maiores índices de incidência solar do território brasileiro, o que o torna apto a receber investimentos para a instalação de usinas solares fotovoltaicas. Este artigo analisará o potencial de geração da energia solar fotovoltaica no RN através de medições solarimétricas realizadas em Natal, capital do estado.

PALAVRAS-CHAVE: Energia Solar Fotovoltaica, Matriz energética, Fontes Alternativas de energia, Geração de Energia Elétrica, Geração Distribuída.

\section{PROSPECTS FOR INTEGRATION OF PHOTOVOLTAIC SOLAR ENERGY IN ELECTRICITY GENERATION IN RIO GRANDE DO NORTE STATE}

\section{ABSTRACT}

The generation of power electricity in Brazil is predominantly renewable generation with internal hydraulic being more than $74 \%$ of its total energy matrix. The electricity rationing occurred in 2001, due to lack of rain, led the country to increase the participation of alternative energy sources. This need for new sources of energy makes the regional potential to be exploited, which configures the change of generation model from centralized generation to distributed generation. Among the alternative sources of energy, solar is presented as very promising for Brazil, given that most of its territory is located near to the equator line, which causes a longer duration of the solar day. The Rio Grande do Norte (RN) has one of the highest levels of solar irradiation of the Brazilian territory, making it eligible to receive investments for the installation of photovoltaic solar plants. This article will examine the potential for generation of solar photovoltaics in RN through solarimetrics measurements performed in Natal, the state capital.

KEYWORDS: Solar Photovoltaic Energy, Energy Matrix, Alternative Sources of Energy, Generation of Electricity, Distributed Generation. 


\section{PERSPECTIVAS DE INSERÇÃO DA ENERGIA SOLAR FOTOVOLTAICA NA GERAÇÃO DE ENERGIA ELÉTRICA NO RIO GRANDE DO NORTE}

\section{INTRODUÇÃO}

A energia solar total incidente sobre a superfície terrestre depende das condições atmosféricas (nebulosidade, umidade relativa do ar etc.), da latitude local e da posição no tempo (hora do dia e dia do ano) [1]. A quantidade anual que a Terra recebe de energia solar corresponde a 10 mil vezes o consumo energético mundial neste período [2].

A energia solar pode ser utilizada como fonte de energia térmica ou pode ser convertida diretamente em energia elétrica através do efeito fotovoltaico.

O Plano Nacional de Energia 2030 (PNE 2030) [3] reproduz dados do Atlas Solarimétrico do Brasil mostrando que essa radiação varia de 8 a $22 \mathrm{MJ} / \mathrm{m}^{2}$ durante o dia, sendo que as menores variações ocorrem nos meses de maio a julho, com essa radiação variando de 8 a 18 $\mathrm{MJ} / \mathrm{m}^{2}$. Este documento informa também que o Nordeste brasileiro é a região de maior radiação solar, apresentando as melhores condições para o aproveitamento fotovoltaico.

Praticamente todas as regiões recebem mais de 2.200 horas de insolação, com um potencial equivalente a 15 trilhões de $\mathrm{MWh}$, correspondentes a 50 mil vezes o consumo nacional de eletricidade [4].

Mesmo com todo este potencial, a energia solar tem pouca participação na matriz energética brasileira, não aparecendo no Balanço Energético Nacional (BEN), edição de 2012 [5].

O Banco de Informações de Geração (BIG) [6] da Agência Nacional de Energia Elétrica (ANEEL) também não faz menção a qualquer usina fotovoltaica.

O Rio Grande do Norte (RN), estado localizado na Região Nordeste do Brasil, destaca-se como tendo um alto índice de radiação solar. Medições solarimétricas realizadas em Natal e apresentadas neste artigo confirmam esse potencial, justificando a implantação de centrais solares fotovoltaicas no Brasil e em particular no RN.

\section{ENERGIA SOLAR FOTOVOLTAICA NO BRASIL}

O Brasil alcançou em 2011 uma capacidade instalada total de geração elétrica igual a 117,135 MW na soma das centrais de serviço público e autoprodutoras, conforme o BEN [5].

A Figura 1 apresenta a estrutura da oferta interna de eletricidade no Brasil em 2011. Observa-se que a matriz de geração elétrica no Brasil é predominantemente renovável, com a geração interna hidráulica correspondendo a $74 \%$ da oferta.

Adicionando a este valor as importações de energia, que também são de origem renovável, o BEN afirma que aproximadamente $89 \%$ da energia elétrica no Brasil são originadas de fontes renováveis.

O uso da energia solar fotovoltaica no Brasil contou com algumas iniciativas voltadas para a eletrificação rural, através de concessionárias e instituições, conforme [7] citado em [8]

- PROGRAMA LUZ SOLAR, implantado no estado de Minas Gerais;

- PROGRAMA LUZ DO SOL, implantado na Região Nordeste e

- Programa Nacional de Eletrificação Rural, LUZ NO CAMPO. 


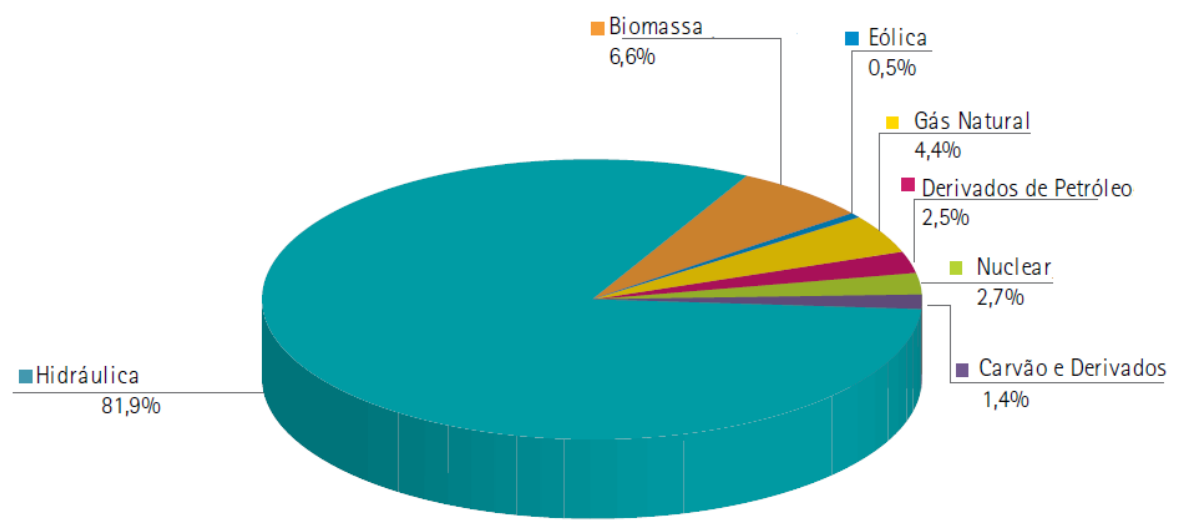

Figura 1 - Oferta Interna de Energia Elétrica por Fonte - 2011 FONTE: [5] (adaptado).

Entretanto, somente com o Programa de Desenvolvimento Energético de Estados e Municípios (PRODEEM), a energia solar fotovoltaica foi incorporada à matriz energética brasileira.

Implantado em 1994 pelo Governo Federal, o PRODEEM foi baseado principalmente na tecnologia fotovoltaica, compreendendo três tipos de aplicações: sistemas fotovoltaicos de geração de energia elétrica, sistemas fotovoltaicos de bombeamento d'água e sistemas fotovoltaicos de iluminação pública.

Nesse programa, instalou-se o equivalente a $5 \mathrm{MWp}$ de sistemas fotovoltaicos em aproximadamente 7.000 comunidades em todo Brasil [9].

O Programa Nacional de Universalização do Acesso e Uso da Energia Elétrica (PROGRAMA LUZ PARA TODOS), instituído em 2003 pelo governo federal e substituto do PROGRAMA LUZ NO CAMPO, incorporou o PRODEEM.

Em 2004 foi criado o Centro Brasileiro para o Desenvolvimento da Energia Solar Fotovoltaica (CB-SOLAR), o qual desenvolve um projeto para a produção industrial de módulos fotovoltaicos de alta eficiência e baixo custo.

Em 2011 entrou em operação no estado do Ceará, região Nordeste, a Usina Solar Fotovoltaica de Tauá, com capacidade de $1 \mathrm{MW}$. É a primeira usina solar fotovoltaica comercial da América Latina e a única conectada ao Sistema Interligado Nacional (SIN).

\section{CONCEITOS BÁSICOS}

A irradiação solar depende de diversos fatores: a localidade, a estação do ano, a composição atmosférica, a cobertura de nuvens e a forma da superfície.

O dimensionamento e o desempenho de uma central fotovoltaica dependem de alguns parâmetros: radiação global, radiação direta, radiação difusa, horas de sol, temperatura média e velocidade do vento.

Como a intensidade da radiação solar é variável, é necessário um levantamento histórico dos seus valores para garantir um melhor aproveitamento do sistema de geração ao longo do ano.

A densidade média do fluxo energético proveniente da radiação solar quando medida num plano perpendicular à direção da propagação dos raios solares no topo da atmosfera terrestre é igual a $1.367 \mathrm{~W} / \mathrm{m}^{2}$, valor este designado por constante solar [2]. 


\section{CÉLULAS FOTOVOLTAICAS}

A utilização da energia solar fotovoltaica oferece uma série de vantagens. Ela é uma fonte ilimitada de energia e está disponível em todas as partes do mundo. Não produz ruídos ou gases nocivos, nem resíduos. Os sistemas fotovoltaicos são fáceis de instalar e praticamente não precisam de manutenção. Os módulos fotovoltaicos têm duração próxima de 30 anos e os sistemas fotovoltaicos são seguros. A energia pode ser gerada em áreas remotas. Os materiais utilizados podem ser reciclados e a indústria para geração solar pode criar milhares de empregos. Os painéis não têm peças móveis, o que exige pouca manutenção e é possível aumentar a potência instalada por meio da incorporação de módulos adicionais.

A energia solar pode ser convertida em energia elétrica através de duas tecnologias: células fotovoltaicas e concentradores de calor. Este artigo abordará as células fotovoltaicas.

As células fotovoltaicas convertem a energia radiante em energia elétrica através do efeito fotoelétrico, que ocorre quando um elétron salta para uma órbita mais externa em função da incidência de luz sobre a célula.

O efeito fotovoltaico consiste no surgimento de uma tensão elétrica entre dois materiais semicondutores de propriedades elétricas diferentes quando incide luz na junção entre os dois.

Para a fabricação de células fotovoltaicas o material mais utilizado é o silício. De acordo com a estrutura de ligação dos seus átomos, as células fotovoltaicas podem ser do tipo cristalina (monocristalina ou policristalina) ou do tipo amorfa.

O silício monocristalino (mono-Si) é obtido a partir de um único cristal imerso em um banho de silício fundido de alto grau de pureza. Devido às perdas de material ocorridas neste processo, torna-se um produto caro. Células fotovoltaicas fabricadas com este tipo de silício têm a melhor eficiência na transformação de energia solar em elétrica, da ordem de $18 \%$ [10]. Em 2011 conseguiu-se uma eficiência de 25\% para esse tipo de célula [11].

O silício policristalino (poly-Si) diferencia-se do monocristalino pelo fato de que é obtido a partir da solidificação do silício fundido. Seu custo de produção é menor e seu rendimento é da ordem de $16 \%$ [10].

O silício amorfo (a-Si) consiste em uma forma de silício não cristalina e células fabricadas a partir dele tem um rendimento da ordem de $10 \%$ [10].

A tecnologia dos filmes finos possibilita a produção de células mais baratas do que as fabricadas com silício cristalino. Entretanto, as células baseadas em filmes finos apresentam rendimento baixo.

A eficiência dos filmes finos varia de acordo com o material empregado na sua fabricação [12]:

- de silício amorfo varia de $4 \%$ a $8 \%$;

- com o telureto de cádmio (CdTe) varia de 10\% a 11\% (em 2011 atingiu-se 17\% [11]) e

- com o Disseleneto de Cobre-Índio-Gálio (CIGS) varia de 7\% a 12\% (em 2013 atingiu-se $19,7 \%[11])$.

O silício amorfo está sendo substituído pelo telureto de cádmio na produção de filmes finos.

\section{MEDIÇÕES SOLARIMÉTRICAS}


Várias iniciativas para mapeamento do potencial solar no Brasil foram feitas por diferentes instituições. Os mais recentes levantamentos para avaliação da radiação solar estão disponíveis nos seguintes atlas ([13] e [14] citados por [15]):

- Atlas de Irradiação Solar no Brasil: Publicado em 1998, foi elaborado pelo Instituto Nacional de Pesquisas Espaciais (INPE) e pelo Laboratório de Energia Solar (LABSOLAR) da Universidade Federal de Santa Catarina (UFSC), em parceria com outras instituições de pesquisa;

- Atlas Solarimétrico do Brasil: Publicado em 2000, foi elaborado pela Universidade Federal de Pernambuco (UFPE) em parceria o Centro de Pesquisas de Energia Elétrica da Eletrobrás (CEPEL), através do Centro de Referência para Energia Solar e Eólica Sérgio de Salvo Brito (CRESESB), e a Companhia Hidroelétrica do São Francisco (CHESF). Várias instituições (secretarias de agricultura, universidades, instituições de pesquisa) colaboraram com as informações contidas no atlas e

- Atlas Brasileiro de Energia Solar: Publicado em 2006 no âmbito do projeto SWERA (Solar and Wind Energy Resources Assessment), foi coordenado pelo INPE em associação com o CEPEL e o LABSOLAR/UFSC.

A Rede SONDA (Sistema de Organização Nacional de Dados Ambientais) é um projeto do Instituto Nacional de Pesquisas Espaciais (INPE) destinado à levantar e melhorar a base de dados dos recursos de energia solar e eólica no Brasil. A rede de Estações SONDA conta com 13 estações próprias e 2 estações colaboradoras distribuídas por todo o território brasileiro [16].

A Rede SONDA tem uma estação solarimétrica instalada em Natal-RN na latitude $5^{\circ} 50^{\prime}$ $12^{\prime \prime}$ Sul e longitude $35^{\circ} 12^{\prime} 23^{\prime \prime}$ Oeste. Esta estação dispõe de sensores para a coleta de dados de radiação solar, realizando medições nas componentes global, direta e difusa, bem como dados sobre iluminância e radiação fotossintética ativa, importante para o setor agrícola [16].

\section{METODOLOGIA UTILIZADA}

As medições solarimétricas utilizadas no presente artigo foram realizadas no Laboratório de Máquinas Hidráulicas e Energia Solar da Universidade Federal do Rio Grande do Norte (UFRN), que em parceria com a Universidade de Coimbra possui um equipamento de aquisição de dados climatológicos.

Este laboratório situa-se a uma altitude de $60 \mathrm{~m}$ em relação ao nível do mar, com latitude $5^{\circ} 50^{\prime} 31^{\prime \prime}$ Sul e longitude $35^{\circ} 11^{\prime} 48^{\prime \prime}$ Oeste.

Os dados obtidos pelo equipamento são transmitidos em tempo real para um computador, o qual processa as informações.

Dentre as diversas propriedades coletadas, podemos citar:

- umidade do ar;

- energia solar;

- radiação solar;

- índice de radiação UV;

- temperatura externa;

- precipitação de chuva e 
- velocidade do vento.

A maioria desses parâmetros é essencial para o dimensionamento de um sistema fotovoltaico.

\section{ANÁLISE DE RESULTADOS}

Este artigo apresenta os dados coletados no período de 26 de fevereiro de 2010, data da instalação do equipamento, até 10 de Julho de 2012, em intervalos de 30 minutos [17].

As Figuras 2 a 4 apresentam os gráficos referentes à radiação solar média medida pelo equipamento no período de fevereiro de 2010 a julho de 2012.

Os valores medidos mostram a alta incidência de radiação solar em Natal-RN e constatase também, através destes gráficos, uma incidência de radiação solar que não apresenta variações extremas ao longo dos meses, em cada ano analisado. Esta é uma condição excelente do ponto de vista de aproveitamento da energia solar para geração de energia elétrica.

Outro importante fator medido pelo equipamento e utilizado no estudo do potencial de geração de energia solar é a precipitação de chuva.

As Figuras 5 a 7 apresentam os gráficos referentes à precipitação de chuva medida pelo equipamento no período de fevereiro de 2010 a julho de 2012, ou seja, no mesmo período representado nas Figuras 2 a 4 acima.

Os gráficos de precipitação de chuva apresentam como característica principal a variação extrema entre os valores mês a mês, prevalecendo valores baixos.

Percebe-se que, mesmo com a variação acentuada no regime de chuvas, praticamente não houve alterações significativas nos valores da radiação solar incidente. Isto é muito importante quando pretende-se aproveitar a radiação solar para gerar energia elétrica.

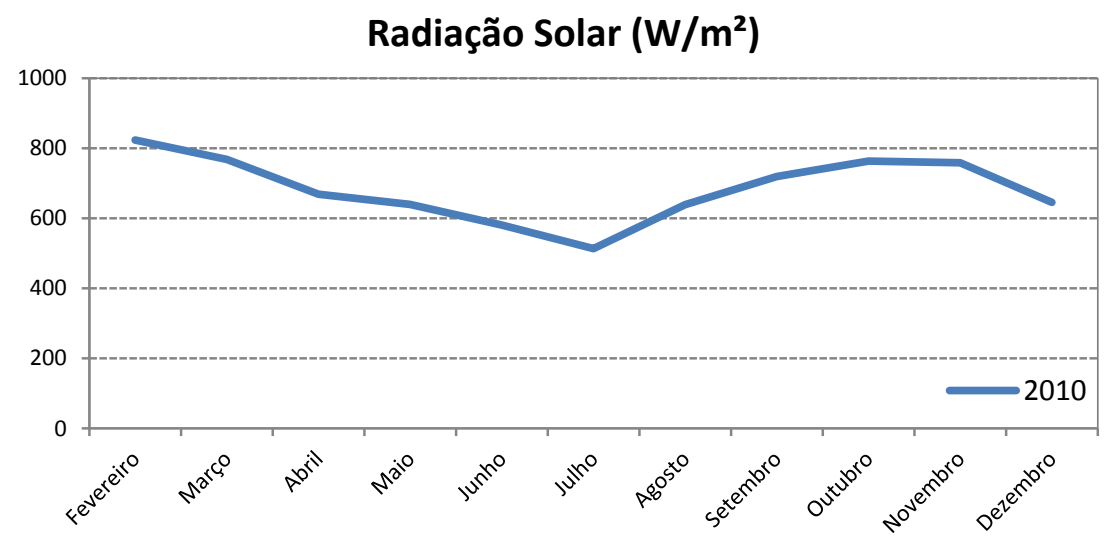

Figura 2 - Gráfico mostrando a radiação solar média em 2010

FONTE: [17]. 


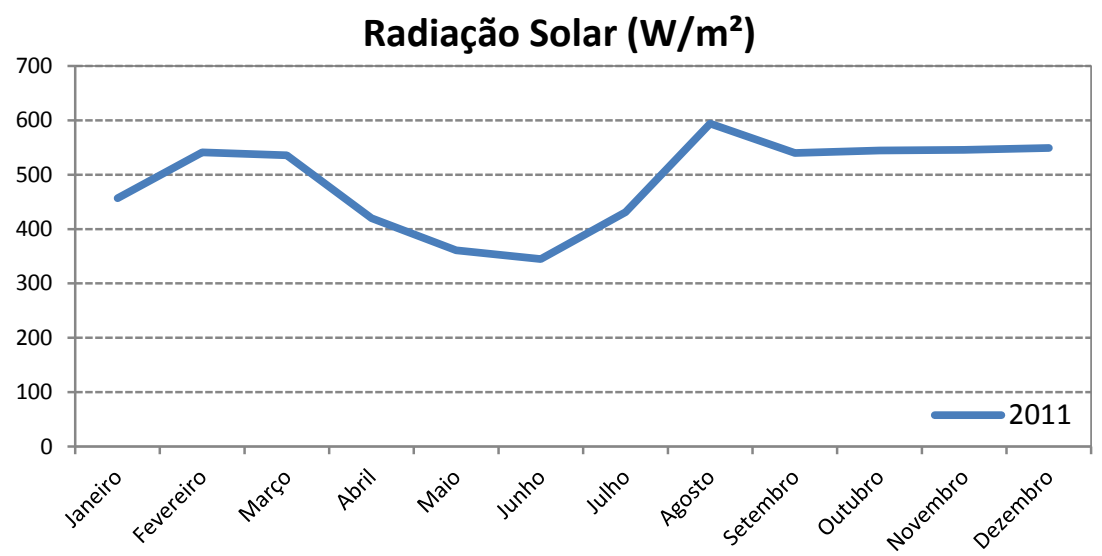

Figura 3 - Gráfico mostrando a radiação solar média em 2011 FONTE: [17].

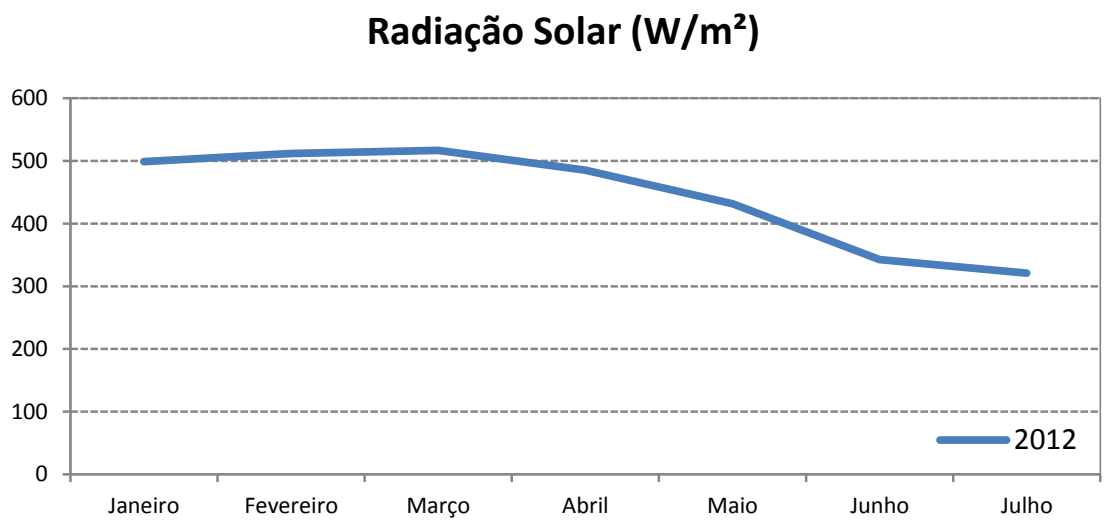

Figura 4 - Gráfico mostrando a radiação solar média em 2012 FONTE: [17]

\section{Precipitação de chuva $(\mathrm{mm})$}

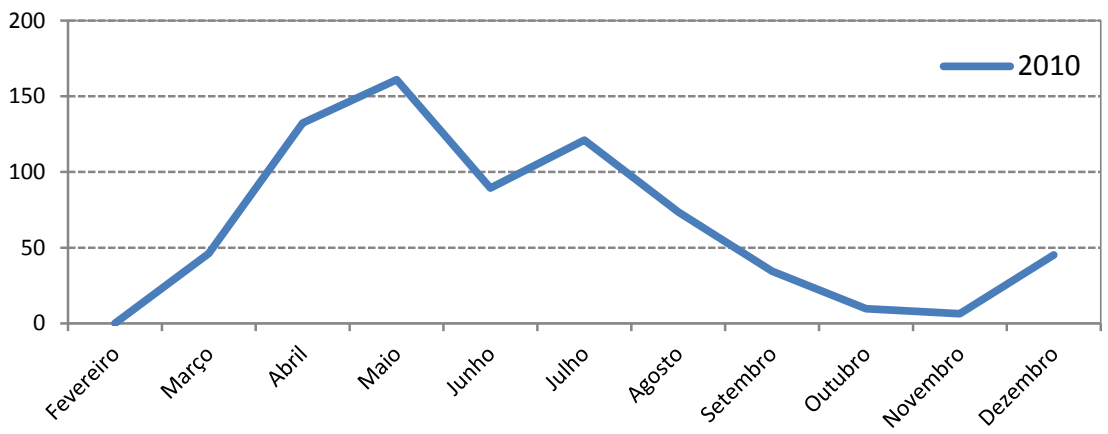

Figura 5 - Gráfico mostrando a precipitação de chuva em 2010 FONTE: [17]. 


\section{Precipitação $(\mathrm{mm})$}

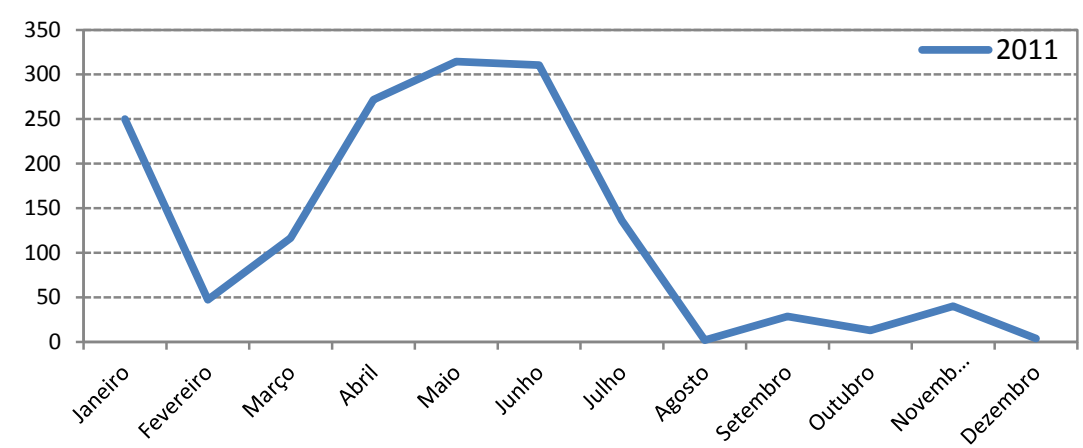

Figura 6 - Gráfico mostrando a precipitação de chuva em 2011 FONTE: [17].

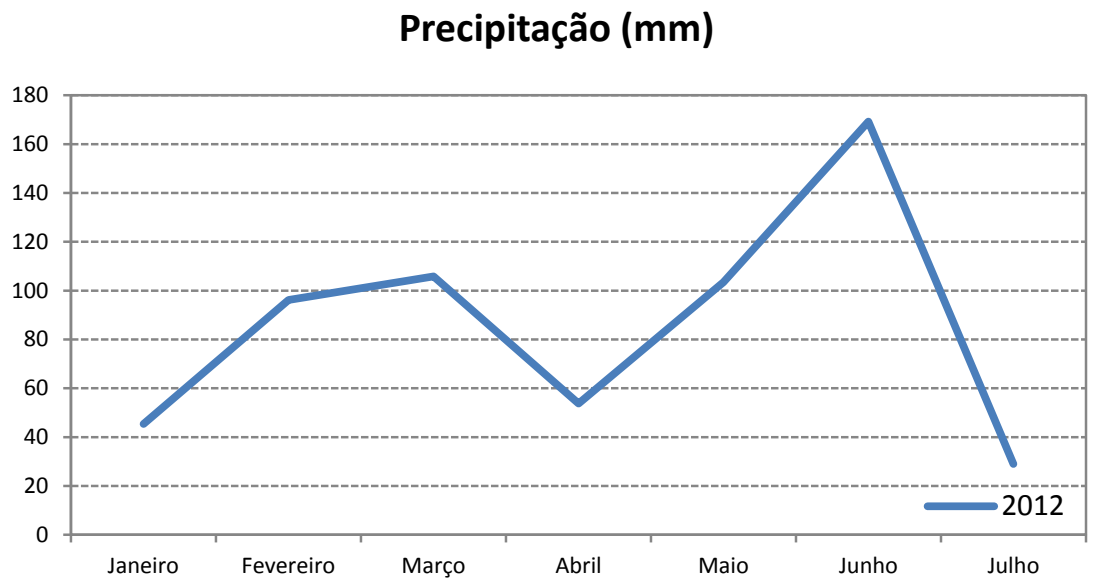

Figura 7 - Gráfico mostrando a precipitação de chuva em 2012 FONTE: [17].

\section{CENÁRIO ATUAL, AVANÇOS E PERSPECTIVAS}

O Plano Nacional de Energia 2030 apresenta a energia solar como uma alternativa para atender a crescente demanda de energia elétrica no país, bem como para manter a participação de renováveis na matriz elétrica.

Iniciativas importantes estão sendo tomadas para regulamentar e disseminar o uso da energia solar fotovoltaica no Brasil.

A ANEEL publicou em agosto/2011 a Chamada de Projeto Estratégico de Pesquisa \& Desenvolvimento № 013/2011 intitulada "Arranjos Técnicos e Comerciais para Inserção da Geração Solar Fotovoltaica na Matriz Energética Brasileira". Foram qualificados 18 projetos, distribuídos em 96 empresas, 62 instituições de ensino e pesquisa e 584 pesquisadores. Os projetos totalizam uma geração de $24,5 \mathrm{MWp}$ no prazo de três anos [18].

Entre os objetivos dessa chamada estão: facilitar a inserção da geração solar fotovoltaica na matriz energética brasileira, estimular a redução de custos da geração solar e incentivar o desenvolvimento no país de toda a cadeia produtiva da indústria solar fotovoltaica.

Outras ações da ANEEL que estimulam o uso da energia solar fotovoltaica são: 
- Resolução № 481/2012, que altera o desconto de 50\% para $80 \%$ nas tarifas de uso dos sistemas de distribuição e transmissão (TUSD e TUST) para usinas com fonte solar para os empreendimentos que entrarem em operação comercial até dezembro de 2017 [19];

- Resolução № 482/2012, que estabelece as condições gerais para o acesso de microgeração e minigeração distribuídas aos sistemas de distribuição de energia elétrica e o sistema de compensação de energia elétrica [20];

- Resolução № 493/2012, que estabelece os procedimentos e as condições de fornecimento por meio de Microssistema Isolado de Geração e Distribuição de Energia Elétrica - MIGDI ou Sistema Individual de Geração de Energia Elétrica com Fonte Intermitente-SIGFI [21].

A Associação Brasileira de Normas Técnica (ABNT) [22] lançou três importantes normas para a área fotovoltaica, que juntamente com às já existentes, permitirão à indústria brasileira tornar-se capaz de fornecer a quase totalidade de uma instalação fotovoltaica:

- NBR IEC 62116:2012 - Procedimento de ensaio de anti-ilhamento para inversores de sistemas fotovoltaicos conectados à rede elétrica;

- NBR 16149:2013 - Sistemas fotovoltaicos (FV) - Características da interface de conexão com a rede elétrica de distribuição e

- NBR 16150:2013 - Sistemas fotovoltaicos (FV) - Características da interface de conexão com a rede elétrica de distribuição - Procedimento de ensaio de conformidade.

O Grupo Setorial de Sistemas Fotovoltaicos (GS-Fotovoltaico) da Associação Brasileira da Indústria Elétrica e eletrônica (ABINEE), composto por mais de 140 empresas, lançou recentemente um estudo com propostas para inserção da energia solar fotovoltaica na matriz elétrica brasileira [23].

O Plano Decenal de Expansão de Energia 2021 [24] afirma que o custo de geração fotovoltaica distribuída já alcançou a paridade com as tarifas na rede de distribuição de algumas concessionárias.

Outra iniciativa importante para a ampliação do uso da energia solar fotovoltaica é a instalação de sistemas fotovoltaicos nos estádios que sediarão os jogos da Copa do Mundo em 2014 [25].

A produção residencial de energia solar já é viável para $15 \%$ dos domicílios brasileiros, cujo custo de geração de um equipamento de pequena potência é de $\mathrm{R} \$ 602,00 / \mathrm{MWh}$, mais barato do que a energia vendida por dez das mais de 60 distribuidoras de energia do país [26].

No RN, está em fase de desenvolvimento o projeto de uma usina a ser instalada no município de Alto do Rodrigues, distante $20 \mathrm{~km}$ de Natal. A planta terá capacidade instalada de 1,1 MW e deverá gerar 1,65 GWh por ano, devendo ser interligada ao Sistema Interligado Nacional (SIN) [27].

Também no Rio Grande do Norte está sendo preparado o edital de licitação pública para elaboração do atlas solarimétrico do estado [28].

A quantidade de empregos gerada pela indústria solar fotovoltaica é bastante significativa nos Estados Unidos. Em 2011, essa indústria gerou 54 empregos diretos por MW instalado. Estudos mostram que empregos gerados pela fonte solar fotovoltaica são superiores às demais fontes e tecnologias [29]. 


\section{CONCLUSÕES}

$\mathrm{O}$ artigo mostrou que avanços importantes foram e estão sendo dados para a implantação e consolidação da energia solar fotovoltaica no Brasil, visando o desenvolvimento da sua cadeia produtiva e de serviços nacional.

Contudo, para expandir o uso da energia solar fotovoltaica na matriz energética do Brasil, devem ser adotadas medidas que se enquadrem nas características do sistema elétrico do país, baseando-se inclusive, nos modelos de sucesso adotados em outros países, principalmente na Alemanha, Japão e Estados Unidos.

Medições de radiação solar realizadas em Natal, capital do RN, e apresentadas neste artigo comprovam o alto potencial para geração de energia elétrica a partir do sol.

Vários investidores, sejam fabricantes de painéis solares ou construtores de usinas fotovoltaicas, têm demonstrado interesse em se instalar no RN.

Políticas fiscais e de financiamento, a consolidação de uma cadeia produtiva, a realização de leilão específico e a pesquisa e inovação tecnológica farão com que a energia solar fotovoltaica desenvolva-se no Brasil e torne-se importante na matriz energética brasileira, de forma a atender as necessidades do país.

\section{AGRADECIMENTOS}

Agradecemos ao professor Luiz Guilherme Meira de Souza, do Departamento de Engenharia Mecânica da Universidade Federal do Rio Grande do Norte, a cessão dos dados solarimétricos que serviram de base para a elaboração deste artigo.

\section{REFERÊNCIAS BIBLIOGRÁFICAS}

1. AGÊNCIA NACIONAL DE ENERGIA ELÉTRICA (BRASIL). Atlas de energia elétrica do Brasil. 3. ed. Brasília: ANEEL, 2008. Disponível em: <http://www.aneel.gov.br/visualizar_texto.cfm?idtxt=1689>. Acesso em: 14 fev. 2013.

2. CENTRO DE PESQUISAS DE ENERGIA ELÉTRICA. CENTRO DE REFERÊNCIA PARA ENERGIA SOLAR E EÓLICA SÉRGIO DE SALVO BRITO. Manual de engenharia para sistemas fotovoltaicos. Rio de Janeiro: CRESESB, 2004. Disponível em: < http://www.cresesb.cepel.br/publicacoes/download/Manual_de_Engenharia_FV_2004.pdf >. Acesso em: 10 mar. 2013.

3. MINISTÉriO DE MINAS E ENERGIA. EMPRESA DE PESQUISA ENERGÉTICA (BRASIL). Plano Nacional de Energia 2030. Brasília: MME: EPE, 2007. Disponível em: < http://www.mme.gov.br/mme/galerias/arquivos/publicacoes/pne_2030/PlanoNacionalDeEn ergia2030.pdf >. Acesso em: 18 mar. 2013.

4. RODRIGUES, Délcio; MATAJS Roberto. Um banho de sol para o Brasil: o que os aquecedores solares podem fazer pelo meio ambiente e a sociedade. São Lourenço da Serra: Vitae Civilis, 2005.

5. EMPRESA DE PESQUISA ENERGÉTICA (BRASIL). Balanço Energético Nacional 2012: Ano base 2011. Rio de Janeiro: EPE, 2012. <https://ben.epe.gov.br/downloads/Relatorio_Final_ BEN_2012.pdf>. Acesso em: 15 fev. 2013. 
6. AGÊNCIA NACIONAL DE ENERGIA ELÉTRICA (BRASIL). Banco de Informações de Geração. Disponível em:

http://www.aneel.gov.br/aplicacoes/capacidadebrasil/operacaocapacidadebrasil.asp > Acesso em: 01 jun. 2013.

7. WINROCK INTERNATIONAL - BRAZIL, 2002. Trade guide on renewable energy in Brazil. October 2002.

8. VARELLA, F., CAVALIERO, C., SILVA, E.. REGULATORY INCENTIVES TO PROMOTE THE USE OF PHOTOVOLTAIC SYSTEMS IN BRAZIL. HOLOS - ISSN 1807-1600, Natal, 3, jun. 2012. Disponível em: <http://www2.ifrn.edu.br/ojs/index.php/HOLOS/article/view/883>. Acesso em: 08 out. 2012.

9. MINISTÉRIO DE MINAS E ENERGIA (BRASIL). Estudo e propostas de utilização de geração fotovoltaica conectada à rede, em particular em edificações urbanas. Brasília: MME, 2009.

10. CENTRO DE PESQUISAS DE ENERGIA ELÉTRICA. CENTRO DE REFERÊNCIA PARA ENERGIA SOLAR E EÓLICA SÉRGIO DE SALVO BRITO. Disponível em: <http://cresesb.cepel.br/content.php?cid=381>. Acesso em: 02 jun. 2013.

11. SILVA, Selênio Rocha. EEE024 - Fundamentos em fontes alternativas de energia. Disponível em: <http://www.cpdee.ufmg.br/ selenios/Geracao/EEE024-2013_3.pdf>. Acesso em: 02 jun. 2013.

12. EMPRESA DE PESQUISA ENERGÉTICA (BRASIL). Análise da inserção da geração solar na matriz elétrica brasileira. Rio de Janeiro: EPE, 2012. Disponível em: < http://www.epe.gov.br/geracao/Documents/Estudos_23/NT_EnergiaSolar_2012.pdf >. Acesso em: 22 jun. 2012.

13. VIEIRA, Leonardo. Eletricidade solar: visão do CEPEL. Workshop sobre usinas solares termelétricas. Recife, 2010.

14. PEREIRA, Enicio Bueno; MARTINS, F.R.; ABREU, S. L.; RUTHER, R. Atlas brasileiro de energia solar. São José dos Campos: INPE, 2006. Disponível em: < http://mtcm17.sid.inpe.br/col/sid.inpe.br/mtc-m17@80/2007/05.04.14.11/doc/atlas_solar-reduced.pdf $>$.

15. LODI, Cristiane. Perspectivas para a geração de energia elétrica no Brasil utilizando a tecnologia solar térmica concentrada. 2011. 127 p. Dissertação (Mestrado em Planejamento Energético)- UFRJ/COPPE, Rio de Janeiro, 2011.

16. INSTITUTO NACIONAL DE PESQUISAS ESPACIAIS. SISTEMA DE ORGANIZAÇÃO NACIONAL DE DADOS AMBIENTAIS (REDE SONDA). Disponível em: <http://sonda.ccst.inpe.br/>. Acesso em: 18 mar. 2013.

17. MEDEIROS, Elvis Néris de. Relatório Final de Estágio. Natal, 2012.

18. AGÊNCIA NACIONAL DE ENERGIA ELÉTRICA (BRASIL). P\&D 013/2011 - Arranjos Técnicos e Comerciais para Inserção da Geração Solar Fotovoltaica na Matriz Energética Brasileira. Disponível em: < http://www.aneel.gov.br/arquivos/PDF/P\&D_Est_013-2011-r2.pdf >. Acesso em: 30 abr. 2013.

19.

Resolução normativa no 481. Disponível em: <http://www.aneel.gov.br/cedoc/ren2012481.pdf>. Acesso em: 20 maio. 2013. 
20. . Resolução normativa no 482. Disponível em: <http://www.aneel.gov.br/cedoc/bren2012482.pdf>. Acesso em: 20 maio. 2013.

21. Resolução normativa no 493. Disponível em: < http://www.aneel.gov.br/cedoc/ren2012493.pdf >. Acesso em: 20 maio. 2013.

22. ASSOCIAÇÃO BRASILEIRA DE NORMAS TÉCNICAS. Normas técnicas. Disponível em: <http://www.abnt.org.br/>. Acesso em: 20 maio. 2013.

23. ASSOCIAÇÃO BRASILEIRA DA INDÚSTRIA ELÉTRICA E ELETRÔNICA. Propostas para inserção da energia solar fotovoltaica na matriz elétrica brasileira. São Paulo: ABINEE, 2012. Disponível em: < http://www.abinee.org.br/informac/arquivos/profotov.pdf >. Acesso em: 30 jul. 2012.

24. MINISTÉRIO DE MINAS E ENERGIA. EMPRESA DE PESQUISA ENERGÉTICA (BRASIL). Plano Decenal de expansão de energia 2021. Brasília: MME: EPE, 2012. Disponível em: <http://www.epe.gov.br/PDEE/20130326_1.pdf>. Acesso em: 15 fev. 2013.

25. UNIVERSIDADE FEDERAL DE SANTA CATARINA. INSTITUTO PARA O DESENVOLVIMENTO DAS ENERGIAS ALTERNATIVAS NA AMÉRICA LATINA. Relatório estádios solares: opção sustentável para a copa 2014 no Brasil. 2004. Disponível em: <http://www.americadosol.org/wpcontent/uploads/2010/08/Estadios-Solares-UFSC-e-Ideal-GTZ-20100501.pdf>. Acesso em: 10 jan. 2013.

26. ENERGIA solar já é economicamente viável para 15\% dos lares brasileiros. Portal Brasil. $\begin{array}{lllll}\text { Brasília, } & 03 & \text { jul. } & 2012 . & \text { Disponível em: }\end{array}$ http://www.brasil.gov.br/noticias/arquivos/2012/07/03/energia-solar-ja-e-economicamenteviavel-para-15-dos-lares-brasileiros-diz-epe >. Acesso em: 20 ago. 2012.

27. SUNEDISON e Petrobras assinam acordo para construir uma das maiores usinas fotovoltaicas do Brasil. Portal Sunedison. São Paulo, 16 mar 2013. Disponível em: < http://www.sunedison.com.br/info/imprensa/sunedison-petrobras-usinas-fotovoltaicasbrasil.html >. Acesso em: 08 abr. 2013.

28. RIO Grande do Norte prepara licitação para atlas solarimétrico. Portal Jornal da Energia. São Paulo, 24 maio 2013. Disponível em: < http://www.jornaldaenergia.com.br/ler_noticia.php?id_noticia=13589\&id_tipo=3\&id_secao= 8\&id_pai=2 >. Acesso em: 25 maio. 2013.

29. ASSOCIAÇÃO BRASILEIRA DA INDÚSTRIA ELÉTRICA E ELETRÔNICA. Energia fotovoltaica. Disponível em: < http://gsfotovoltaico.com.br/?page_id=66 >. Acesso em: 02 jun. 2013. 